\title{
Virus-Induced Vasculitis
}

\author{
Hisham Sharlala, $M D$, and Ade Adebajo, $M D$
}

\author{
Corresponding author \\ Ade Adebajo, MD \\ Rheumatology Department, Barnsley Foundation Hospital NHS \\ Trust, Barnsley, S75 2EP and Academic Rheumatology Group, \\ University of Sheffield Medical School, Sheffield, S10 2RX, South \\ Yorkshire, United Kingdom. \\ E-mail: a.o.adebajo@sheffield.ac.uk
}

Current Rheumatology Reports 2008, 10:449-452

Current Medicine Group LLC ISSN 1523-3774

Copyright (C) 2008 by Current Medicine Group LLC

There is a growing understanding of the different syndromes that have a definite, and in some cases a possible, association with viral infections. Hepatitis $C$ virus-associated mixed cryoglobulinemias and hepatitis B virus-associated polyarteritis nodosa are examples of a vasculitis with a definite viral association. However, various types of cutaneous vasculitis are examples of a vasculitis with only a possible association with a viral infection.

\section{Introduction}

The link between viral infections and the different vasculitic syndromes has been the subject of discussion for a number of years. With some types of vasculitis, viral infections are thought to be the cause of the disease, whereas with others they may be considered the trigger of an immunologic process of vasculitis followed by the maintenance of the disease process by other factors. However, other vasculitic conditions are thought to be linked to viral infections because of a similarity with viral illness with respect to the age and the time of onset of the disease even though no definite organism has been identified. In this article, we discuss current knowledge with regard to the etiology, epidemiology, pathology, and the possible treatments of viral-induced vasculitis.

\section{Hepatitis C Virus-Associated Cryoglobulinemia} Cryoglobulinemia (CG) is a disorder that can present with variable severity, ranging from the asymptomatic detection of cryoglobulins in the peripheral blood to severe cases of life-threatening, multiorgan small-vessel vasculitis.

Symptomatic cases of CG usually present with weakness, arthralgia, and purpura. Laboratory findings include the presence of serum cryoglobulins, rheumatoid factor, and hypocomplementemia.
Cryoglobulins have been observed in a variety of conditions, such as malignancies, autoimmune diseases, and infections. A viral etiology for CG had been suspected for a long time, and clear evidence of an association with hepatitis $\mathrm{C}$ virus (HCV) infection was described in the 1990s. Most of the essential mixed (no cause identified) CG cases are believed to be caused by HCV infection.

\section{Influence of HCV genotype on} the expression of CG symptoms

There are six types of HCV genotypes $(1,2,3,4,5$, and 6). A study of $340 \mathrm{HCV}$ patients with CG identified $73 \%$ of the cases as asymptomatic CG and $27 \%$ as presenting with CG symptoms. The main risk factor for CG symp-

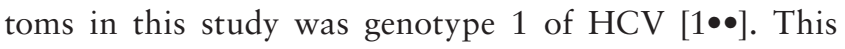
appears to support the previous finding by Gad et al. [2], who compared a cohort of HCV patients from Egypt with genotype 4 (the predominant HCV genotype in Egypt) with another from Japan with genotype $1 \mathrm{~b}$ (the predominant genotype in Japan). They found that the Japanese patients had a significantly higher prevalence of cryoglobulins and symptomatic CG.

\section{Pathophysiology of HCV-associated CG}

Cryoglobulins develop in HCV-infected patients as a result of virus-host interactions. Expansion of rheumatoid factor synthesizing B cells is the histologic hallmark of $\mathrm{HCV}$-associated CG.

The evidence from recent studies shows that high intrahepatic viral load correlates well with the B-cell clonal expansion, in particular the CD5 B-cell fraction in the liver [3]. Other studies have shown a significant enrichment of HCV RNA in the peripheral blood lymphocytes of mixed CG patients compared with a non-mixed CG group, although the exact mechanism of this enrichment in the mixed CG group of patients is not yet clearly understood [4].

\section{HIV coinfection with HCV and}

\section{the association with vasculitis}

HIV infection has been implicated in the etiology of CG. To study the clinical characteristics of HIV-associated CG, Saadoud et al. [5] compared 11 HIV/HCV-coinfected patients with mixed CG vasculitis with $118 \mathrm{HCV}$-infected patients with mixed CG vasculitis and found that both groups had similar clinical manifestations of CG vasculitis, including polyneuropathy, purpura, arthralgia, and 
kidney involvement. However, they also found that the HIV/HCV-coinfected group had significantly lower levels of cryoglobulins in the blood [5].

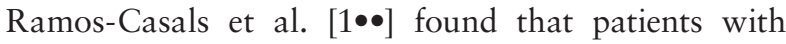
HIV/HCV coinfection, had a significantly lower prevalence of cryoglobulinemic and vasculitic symptoms compared with patients with HCV monoinfection. They concluded that coinfection with HIV attenuated the symptomatic expression of CG in $\mathrm{HCV}$-associated vasculitis. However, a specific subset of HIV/HCV CG patients who had high viral loads of both viruses had a significantly higher frequency of symptomatic CG. This difference in the prevalence of CG symptoms in these groups may simply be due the differentiated HCV genotypes in the different groups, where strong evidence of link between HCV genotype 1 and CG symptoms has already been shown; however, other factors may also play a role $[1 \bullet \bullet]$.

Geographic factors may contribute to the varying expression of rheumatologic conditions, including vasculitis and $\mathrm{CG}$, in $\mathrm{HIV}$ and $\mathrm{HCV}$ viral infections. Zhang et al. [6•] studied 98 consecutive inpatients with HIV infection in China and found that $54 \%$ of them had rheumatologic manifestations. Unlike other studies from other geographic areas, vasculitis was the most common finding (20 cases), including 15 cases of Behcet's-like disease, 2 cases each of Henoch-Schonlein purpura (HSP) and digital gangrene, and one case of central nervous system vasculitis. In this study, CD4 T-cell depletion, erythrocyte sedimentation rate, and HCV coinfection, were the predisposing factors for HIV patients to develop rheumatologic manifestations. It was not clear whether cryoglobulins were tested for in these patients, especially in those patients who had the HSP or digital infarcts presentations [6•].

\section{Treatment of HCV cryoglobulinemia}

Treatment of HCV-associated CG depends on the severity of the presentation. Low doses steroids may be sufficient in controlling the symptoms of arthralgia, weakness, and purpura. In more severe cases, especially where significant renal involvement is present, the aim of treatment is to suppress the viral replication and eliminate the circulating immune complexes.

The use of the combination of standard interferon alpha and ribavirin has been successful in halting the viral replication process and suppressing disease manifestations, although recurrence of the symptoms can also be high with these medications.

The use of pegylated interferon with ribavirin has shown better results than standard interferon with ribavirin. Cacoub et al. [7] have shown in a series of nine patients treated with pegylated interferon and ribavirin that most of their patients achieved complete clinical response, correlating with the eradication of HCV. The treatment duration in this series was shorter than previously reported for standard interferon plus ribavirin [7].
Sansonno et al. [3] observed complete clinical response with the anti-CD20 monoclonal antibody, rituximab, in about $80 \%$ of patients treated for mixed CG vasculitis. In patients who present with the more severe disease, such as acute nephritic syndrome, a more aggressive approach using pulse corticosteroids and cyclophosphamide with plasmapheresis is recommended.

Relapses of mixed CG vasculitis are known to occur in patients with HCV infections, despite achieving sustained viral response. Relapses are usually short lived and may be triggered by the withdrawal of interferon alpha therapy. However, in patients with persistent symptoms of mixed CG vasculitis, different underlying conditions must be considered and looked for, especially B-cell lymphomas. [8•].

\section{Hepatitis B Virus-Associated Vasculitis}

Hepatitis B virus (HBV)-associated vasculitis is an immunologically mediated disorder with clinical manifestations identical to polyarteritis nodosa. It is a severe multisystem disease with positive serum hepatitis B surface antigen. A biopsy of the affected organs shows microaneurysms and corkscrewing of the medium-sized arteries, especially the renal and mesenteric systems.

$\mathrm{HBV}$-associated vasculitis is thought to be a complication of acute HBV infection (characterized by the formation of immune complexes) containing $\mathrm{HB}$ surface antigen and anti-HB surface antibody in the basement membrane of the involved arteries.

\section{Epidemiology}

The highest annual incidence of HBV-associated vasculitis in the world was reported in the 1970s and early 1980s in Alaska, at a rate of 77 cases per million people. Mass vaccination for susceptible people was conducted between 1983 and 1988, which resulted in a significant reduction in the incidence of symptomatic $\mathrm{HBV}$ infections and almost the complete disappearance of HBV-associated vasculitis.

Alaskan natives chronically infected with $\mathrm{HBV}$ have been followed-up twice yearly since 1984 and no new cases of $\mathrm{HBV}$-associated vasculitis have been identified since 1989, which supports the theory that HBV-associated vasculitis is a complication of acute infection and that mass vaccination prevents new cases from occurring $[9 \bullet \bullet]$.

\section{HBV genotypes and the risk of vasculitis}

Eight genotypes of HBV have been identified (A, B, C, D, E, F, G, and H). To identify the specific HBV genotype with the highest association with vasculitis, Hurlburt

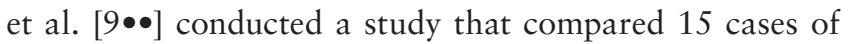
$\mathrm{HBV}$-associated vasculitis and $644 \mathrm{HBV}$-infected controls without vasculitis. They showed that HBV genotype D was more common in $\mathrm{HBV}$-associated vasculitis patients than the controls (odds ratio of 5.9). The results were statistically significant $(P<0.015)$. The authors concluded 
that $\mathrm{HBV}$-associated vasculitis was clearly associated with HBV genotype D.

\section{Treatment of HBV-associated vasculitis}

Before the establishment of the link with HBV infection, treatment of these vasculitis patients included the use of steroids with or without cyclophosphamide, depending on the severity of the presentation.

Since 1983, the treatment of polyarteritis nodosa patients with HBV markers has been with the use of short courses of steroids to suppress the inflammation combined with the use of an antiviral agent (vidarabine, interferon alpha, and lamivudine) to control the viral replication and achieve $\mathrm{HB}$ e antigen to $\mathrm{HB}$ e antibody seroconversion, and with the use of plasma exchange, to clear the circulating immune complexes.

Guillevin et al. [10] has shown a 0\% relapse rate of vasculitis among these patients who achieved $\mathrm{HB}$ e antigen to $\mathrm{HB}$ e antibody seroconversion with the protocol using short courses of steroids, antiviral agent, and plasma exchange. The 7-year overall survival rate was $83 \%$.

\section{Viral-Induced Cutaneous Vasculitis}

Up to $22 \%$ of all cases of cutaneous vasculitis will have a strong association with infective organisms; of these, viral infections contribute a significant proportion. The majority of forms of viral-induced cutaneous vasculitis have a benign self-limiting course, but some will have associated systemic multiorgan involvement with a variable severity and prognosis.

\section{Viral Hepatitis and Cutaneous Vasculitis}

Viral hepatitis is a common cause of cutaneous vasculitis. Eight percent of leukocytoclastic vasculitis (LCV) cases are associated with HCV infection, and 2\% to $4 \%$ of HCV-infected patients develop LCV, usually in association with CG, although LCV has also been reported in the absence of CG [11•].

\section{Henoch-Schonlein Purpura and Viral Infection} The complete tetrad of organ involvement in HSP includes skin, joints, gastrointestinal tract, and kidney. Purpura is universal to HSP. A preceding viral or bacterial upper respi-

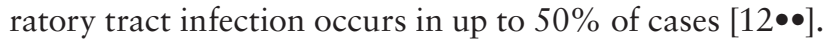

\section{Cutaneous Leukocytoclastic Angiitis and Viral Infection}

In the majority of cases of cutaneous leukocytoclastic angiitis (CLA), no cause is identifiable, but in some cases viral upper respiratory tract infections have been implicated. The skin histology of infection-triggered CLA is likely to show a greater frequency of intraepidermal and epidermal neutrophilic pustules, tissue neutrophilia, prominent IgA vascular deposits, and have relatively less eosinophils and lymphocytes compared with the drug-

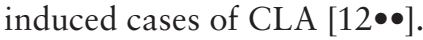

It is important to exclude systemic vasculitis in patients presenting with cutaneous vasculitis before considering the possibility of a self-limiting viral-induced CLA. Histologic clues do sometimes exist to implicate systemic vasculitis, such as palisaded and neutrophilic granulomatous dermatitis (eg, Churg-Strauss syndrome), but in most cases both systemic and benign viral-induced self-limiting CLA manifest themselves in the skin as palpable purpura with identical skin histology. Careful correlation of history, a physical examination, and laboratory findings are required to differentiate localized cutaneous disease from systemic multiorgan vasculitis.

\section{Miscellaneous Vasculitic Conditions with a Possible Link to Viral Infections \\ Kawasaki disease}

Kawasaki disease $(\mathrm{KD})$ is an acute multisystem vasculitis with an unknown etiology affecting mainly young children. Clinical and epidemiologic studies have suggested that $\mathrm{KD}$ is closely related to an infectious disease. $\mathrm{KD}$ is rare in children under 6 months and over 5 years of age. The condition presents with an acute onset, has a self-limiting course, and often occurs in a cluster of cases, which would all suggest that this condition is closely related to a possible viral infection.

Epstein-Barr virus is known to be prevalent in the same age groups that are affected by KD. However, Lee et al. [13] studied the link between Epstein-Barr virus and $\mathrm{KD}$ and concluded that there was no specific association between the two conditions.

Similarly, a blinded case-control study to ascertain the relationship between human corona virus NL63 and KD failed to demonstrate any association [14]. Rowley et al. [15] have described the identification of cytoplasmic inclusion bodies in the ciliated bronchial epithelium of acute KD patients.

IgA plasma cells infiltrate inflamed coronary artery wall in KD. IgA plasma cells are also present in the upper respiratory tract in a similar pattern to that seen in severe viral infections, such as respiratory syncytial or influenza virus infection. The IgA plasma cell infiltration in $\mathrm{KD}$ is suggestive of an immune response to an intracellular pathogen with a respiratory portal of entry.

Further studies of the antigens in the cytoplasmic inclusion bodies suggested the presence of both protein and nucleic acid within the inclusions; no structures resembling bacterial, fungal, or parasitic elements were observed. These studies concluded that the inclusion bodies are most consistent with aggregates of viral proteins and associated nucleic acid. This observation now provides a good direction for future study of the etiology of KD [16•]. 
Varicella zoster virus-related vasculopathy

Varicella zoster virus (VZV) vasculopathy is an increasingly reported central nervous system vasculitis/vasculopathy, occurring secondary to VZV infection. Presentation is typically with a rash, central nervous system vascular event, and the presence of cerebrospinal fluid pleocytosis. Nagel et al. [17] reviewed 14 cases of VZV vasculopathy and found that $100 \%$ of the cases had VZV IgG antibody in the cerebrospinal fluid, whereas only $28 \%$ of the cases had a positive polymerase chain reaction test for VZV DNA in the cerebrospinal fluid. They concluded that testing cerebrospinal fluid for VZV IgG antibodies is the ideal screening investigation for VZV vasculopathy. They also suggested doing the test for cases of central nervous system vasculitis of unknown etiology.

\section{Conclusions}

The evidence for associations between viral infections and certain types of vasculitis is increasing. The association seems to be strongest in conditions like HCV-associated mixed CG and HBV-associated polyarteritis nodosa. The association of specific virus genotypes with symptomatic diseases, such as genotype 1 of HCV and genotype D of HBV, explains, to some extent, why only a specific subgroup of HCV-infected patients develops CG and a specific subset of $\mathrm{HBV}$-infected patients develops polyarteritis nodosa.

Research is ongoing to identify the viral infections associated with other vasculitic conditions such as KD. This should improve the prognosis of these conditions with the future development of targeted treatment and possibly preventive measures, such as the effect seen with mass vaccination for $\mathrm{HBV}$ on the incidence of $\mathrm{HBV}$-associated polyarteritis nodosa.

\section{Disclosures}

No potential conflicts of interest relevant to this article were reported.

\section{References and Recommended Reading}

Papers of particular interest, published recently, have been highlighted as:

- Of importance

$\bullet \quad$ Of major importance

1.• Ramos-Casals M, Forns X, Brito-Zeron P, et al.: Cryoglobulinaemia associated with hepatitis $\mathrm{C}$ virus: influence of $\mathrm{HCV}$ genotype, HCV-RNA viraemia and HIV coinfection. J Viral Hepatitis 2007, 14:736-742.

This is an excellent study that highlights the interesting effect of a specific HCV genotype and HIV coinfection on the expression of symptoms in cryoglobulinemia associated with hepatitis $\mathrm{C}$ virus.

2. Gad A, Tanaka E, Matsumoto A, et al.: Factors predisposing to the occurrence of cryoglobulinaemia in two cohorts of Egyptian and Japanese patients with chronic hepatitis $\mathrm{C}$ infection and genotypic influence. J Med Virol 2003, 70:594-599.
3. Sansonno D, Dammaco F: Hepatitis C virus, cryoglobulinaemia, and vasculitis: immune complex relations. Lancet Infect Dis 2005, 5:227-237.

4. Sansonno D, Lauletta G, Montrone M, et al.: virologic analysis and phenotypic characterization of peripheral blood lymphocytes of hepatitis $\mathrm{C}$ virus-infected patients with and without mixed cryoglobulinaemia. Clin Exp Immunol 2005, 143:288-296.

5. Saadoud D, Aaron L, Resche-Rigon M, et al.: Cryoglobulinaemia vasculitis in patients coinfected with HIV and hepatitis C virus. AIDS 2006, 20:871-877.

6. Zhang X, Li H, Li T, et al.: Distinctive rheumatic manifestations in 98 patients with HIV infection in China. $J$ Rheumatol 2007, 34:1760-1764.

This is a good epidemiologic study describing the distinctive rheumatologic manifestations of HIV-infected and HCV-infected Chinese patients.

7. Cacoub P, Saadoun D, Limal N, et al.: Pegylated interferon alfa- $2 b$ and ribavirin treatment in patients with hepatitis C virus-related systemic vasculitis. Arthritis Rheum 2005, 52:911-915.

8.• Landau D, Saadoun D, Halfon P, et al.: Relapse of hepatitis $C$ virus-associated mixed cryoglobulinaemia vasculitis with sustained viral response. Arthritis Rheum 2008, 58:604-611.

This article provides interesting case reports of relapses among patients with HCV-associated CG. It describes the pattern of these relapses and discusses the differential diagnoses, including lymphoma, especially when presenting atypically.

9.•• Hurlburt K, McMahon B, Simonetti J, et al.: Hepatitis $\mathrm{B}$-associated vasculitis in Alaska natives: viral genotype, clinical and serological outcome. Liver Int 2007, 627-632. This study shows the clear association between genotype $\mathrm{D}$ of the hepatitis B virus and vasculitis. It also demonstrates a significantly positive impact of mass vaccination for hepatitis $B$ on the incidence of the disease.

10. Guillevin L, Maher A, Callard P, et al.: Hepatitis B virusassociated polyarteritis nodosa, clinical characteristics, outcome, and impact of treatment in $\mathbf{1 1 5}$ patients. Medicine 2005, 84:313-322.

11. Alkhatis BA, Adler D: Cutaneous necrotising vasculitis associated with hepatitis C virus infection. Dig Dis $\mathrm{Sci}$ 2007, 52:3438-3439.

This is an interesting case presentation of cutaneous vasculitis in an HCV-infected patient without any evidence of cryoglobulinemia.

12.• Carlson J, Chen K: Cutaneous vasculitis update: small vessel neutrophilic vasculitis syndromes: Am J Dermatopathol 2006, 28:484-506.

This is an important review of cutaneous vasculitis and its link to other clinical syndromes.

13. Lee S, Lee K, Han J, et al.: Epstein-Barr virus antibodies in Kawasaki disease. Yonsei Med J 2006, 47:475-479.

14. Dominguez S, Anderson M, Mary P, et al.: Blinded casecontrol study of the relationship between human corona virus NL63 and Kawasaki syndrome. J Infect Dis 2006, 194:1697-1701.

15. Rowley A, Shulman S, Garcia F, et al.: Cloning the arterial IgA antibody response during acute Kawasaki disease. J Immunol 2005, 175:8386-8391.

16. Rowley A, Shulman S: New developments in the search for the etiologic agent of Kawasaki disease: Curr Opin Pediatr 2007, 19:71-74.

This illuminating article describes the fascinating intracellular changes found in patients with acute KD. These intracellular changes give credence to the possibility of an etiologic link between $\mathrm{KD}$ and viral infections.

17. Nagel M, Forghani B, Mahalingam R, et al.: The value of detecting anti-VZV IgG antibody in the CSF to diagnose VZV vasculopathy: Neurology 2007, 68:1069-1073. 